\title{
Unusual Features of Clear Cell Adenocarcinoma of the Female Urethra: A Case Report and Literature Review
}

\author{
Jianfeng Zeng ${ }^{\mathrm{a}}$, Shanyun Wang ${ }^{\mathrm{b}}$, Liang Zhong ${ }^{\mathrm{a}}$, Zhifeng Huang ${ }^{\mathrm{a}}$, Ye Zeng ${ }^{\mathrm{a}}$, \\ Song $\mathrm{Wu}^{\mathrm{a}}$, Dongxiang Zheng ${ }^{\mathrm{a}}$, Weiwei Zou ${ }^{\mathrm{c}}$, Haibiao Lai ${ }^{\mathrm{a}, \mathrm{d}}$
}

\begin{abstract}
Background: There has been scarce information about clear cell adenocarcinoma of the urethra (CCAU), an extremely rare type of cancer. A few case reports show that CCAU tends to have similar clinical manifestation to the other urethral carcinomas, urethrocystoscopy can easily identify tumoral lesions in the urethra, and bloody drainage from the urethral meatus is often seen during physical exams.
\end{abstract}

Methods: A 52-year-old woman presented with dysuria for 6 months. Urethrocystoscopy did not reveal abnormality, and there was no bloody vaginal drainage or bloody drainage from the urethral meatus during physical exams. Ultrasonography demonstrated a solid mass with well-defined margins located between the anterior vaginal wall and the posterior urethral wall. Computed tomography showed the mass with smooth margins at the level of vaginal fornix, and magnetic resonance imaging showed the same location of the tumor as ultrasonography.

Results: Surgical removal of the tumor was successfully performed and histological and immunohistochemical analysis confirmed the final diagnosis of CCAU.

Conclusion: Abnormality in urethrocystoscopy and bloody drainage during the physical exam were not found in this case, which is in contrast to the findings reported in literature. These unusual features add new knowledge about CCAU that deserves dissemination for improved CCAU diagnosis and management.

Keywords: Case report; Clear cell adenocarcinoma; Urethra; Unu-

Manuscript submitted May 31, 2018, accepted June 4, 2018

${ }^{a}$ Department of Urology Surgery, Hospital of Traditional Chinese Medicine of Zhongshan affiliated with Guangzhou University of Chinese Medicine, Zhongshan City, China

bepartment of Obstetrics and Gynecology, Hospital of Traditional Chinese Medicine of Zhongshan affiliated with Guangzhou University of Chinese Medicine, Zhongshan City, China

'Department of Anesthesiology, Zhongshan Torch Development Zone Hospital, Zhongshan City, China

${ }^{\mathrm{d}}$ Corresponding Author: Haibiao Lai, Department of Urology Surgery, Hospital of Traditional Chinese Medicine of Zhongshan affiliated with Guangzhou University of Chinese Medicine, 3 Kangxin Road, Western District, Zhongshan City 528400, Guangdong Province, China.

Email: laihaibiao@hotmail.com

doi: https://doi.org/10.14740/jocmr3500w sual features

\section{Introduction}

Clear cell adenocarcinoma of the urethra (CCAU), an extremely rare type of cancer, has been reported to occur more commonly in the female than in male [1]. Since Tiltman described the first case of female CCAU in 1975 [2], the information on CCAU remains scarce except that a few case reports have revealed that urethrocystoscopy can easily identify tumoral lesions in the urethra [3-8], and bloody drainage from the urethral meatus is often seen during physical exams [3, 5-7]. The clinical presentation of CCAU is generally similar to those of urethral carcinomas, and final diagnosis relies on histological and immunohistochemical analysis [1]. In this article, we present a case of CCAU in a female patient with unusual features in urethrocystoscopy and physical exams.

\section{Case Report}

A 52-year-old woman who presented with dysuria for 6 months was admitted to our nephrology department in May 2015. Urethrocystoscopy did not reveal abnormality, and ultrasonography demonstrated a solid mass with well-defined margins between the anterior vaginal wall and the posterior urethral wall (Fig. 1a). Computed tomography (CT) showed that the mass was at the level of vaginal fornix and had smooth margins (Fig. 1b), and magnetic resonance imaging (MRI) (Fig. 1c) also showed the same location of the tumor as ultrasonography did. The patient had hysterectomy due to uterine fibroids in 2014. In view of this fact and the findings of urethrocystoscopy and imaging exams, the patient was transferred to the department of obstetrics and gynecology. Pelvic palpation uncovered the mass on the anterior aspect of the vaginal duct and there was no bloody vaginal drainage or bloody drainage from the urethral meatus. The patient underwent surgery and gross examination revealed complete surgical resection of the tumor. The tumor had a size of $4 \mathrm{~cm} \times 3 \mathrm{~cm} \times 3$ $\mathrm{cm}$ with gray white in color, the cut surface of the resected tumor was like rotting flesh, and the tumor was found to be originated from the periurethral tissue. Hobnail-shaped tumor cells were seen in hematoxylin-eosin (HE) staining, and tumor 

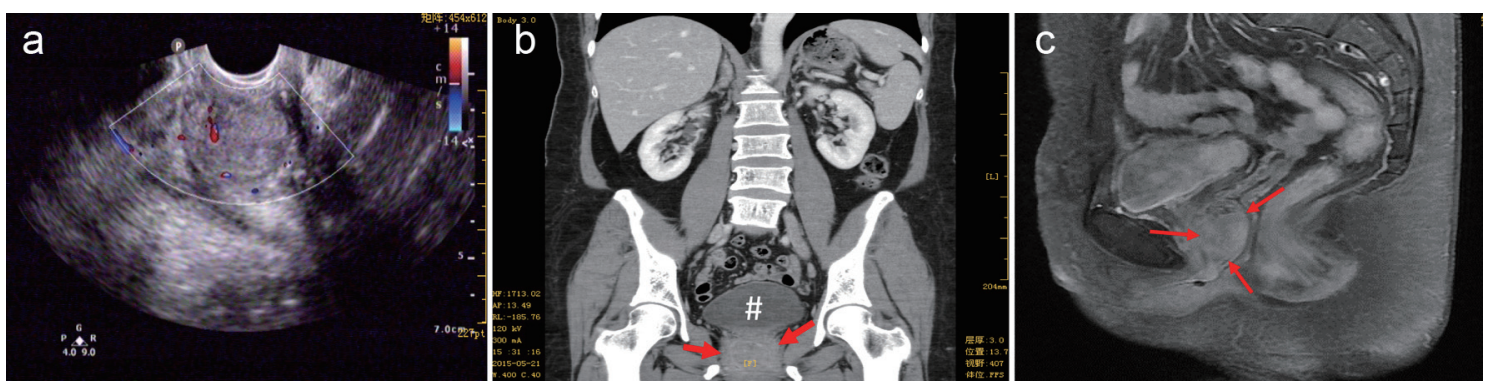

Figure 1. Imaging findings. (a) A hypoechoic mass (about $31 \times 26 \mathrm{~mm}$ ) with well-defined margins between the anterior vaginal wall and the posterior urethral wall was seen by the ultrasound exam. (b) CT scan (feet first-supine position) demonstrated the mass (indicated by the red arrows) was inferior to the urinary bladder (oval-shaped as indicated by \#) approximately the level of vaginal fornix and had smooth margins; (c) the sagittal magnetic resonance T1-weighted imaging showed the tumor (indicated by the red arrows) located at the anterior vaginal wall.

cells had clear cytoplasm without prominent nucleoli (Fig. 2a). Main immunohistochemical findings were as follows: positive staining was found for CK7 (Fig. 2b), Ki-67 and EMA (data not shown); and negative staining for CK5/6, CK20 and P63 (data not shown). The patient was discharged 3 weeks postsurgery. In January 2016, the patient experienced dysuria again. CT and MRI examinations revealed malignant lesions affecting the bladder and vagina. The patient had an anterior pelvic exenteration. Five resected internal iliac lymph nodes were sent for histological and immunohistochemical analysis, which confirmed the recurrence of tumor. In June 2016, the patient was re-admitted into hospital because of pulmonary infection. Chest CT showed multiple pulmonary nodules of varying sizes consistent with metastatic disease. Wide-spread pulmonary embolism in main pulmonary artery and right and left branches was detected by chest CT angiography. Antibiotics and anticoagulation therapy were provided, but the patient eventually died of respiratory failure. During the entire period of treatment, chemotherapy and radiotherapy were declined.

\section{Discussion}

The main complaint by the patient in this study was dysuria, which is in accordance with previous descriptions that dysuria and/or hematuria are the primary initial symptoms encountered in patients with CCAU [1]. Urethrocystoscopy did not reveal abnormality in this case, which is against the findings of other case reports describing easy discovery of the tumor in the urethra with urethrocystoscopy [3-8]. The clinical exam failed to show bloody drainage from the urethral meatus, a feature commonly seen in CCAU patients [1]. These failures are probably due to the fact that the tumor was originated from the periurethral tissue that has been suggested as a possible origin of CCAU, although the histogenesis of CCAU has not been completely resolved $[9,10]$.

Ultrasonography, CT scan and MRI are not confirmatory exams for the diagnosis of CCAU, although Kim et al reported that in MRI images CCAU had a lower height-to-width ratio (range: 0.74 - 1.09) than non-adenocarcinoma (1.25 - 1.88) and non-clear cell adenocarcinoma (1.07 - 1.93) [11]. Our MRI did show an approximate height-to-width ratio of 1.0 (Fig. 1b). However, results by Kim et al were obtained merely from six cases of CCAU; validation of these findings in a larger series remains to be completed, which, however, will be a great challenge in view of the extreme rarity of CCAU. Another interesting MRI finding reported in a case of female CCAU by Sadahira et al is that the CCAU surrounds the urethra as the prostate does in men, suggesting that this characteristic might also be useful in the diagnosis of CCAU [7]. Nevertheless, the final

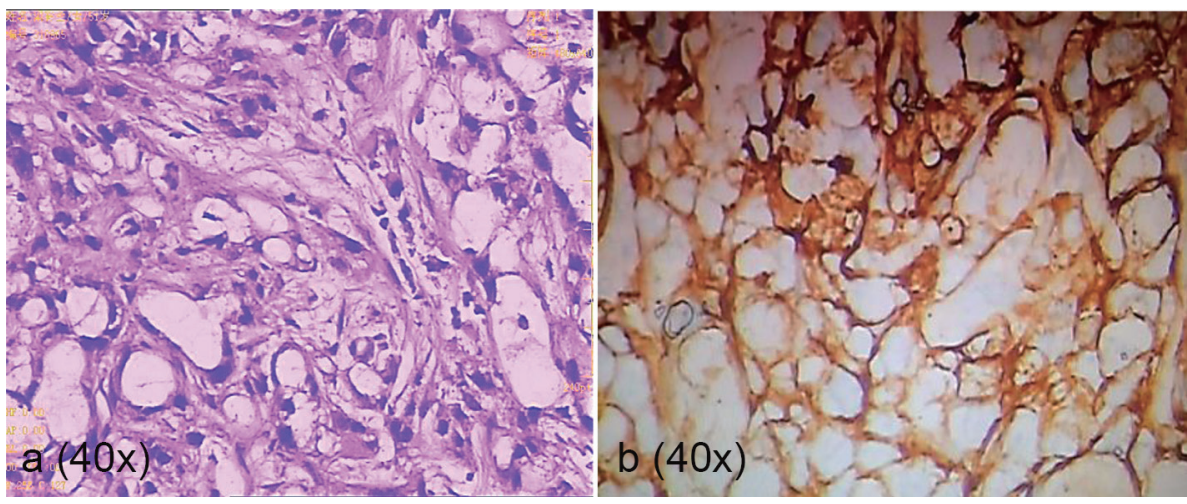

Figure 2. Histology and immunohistochemistry exams. Panel a is a representative image of HE staining that shows numerous tumor cells with clear cytoplasm, a key feature of CCAU. Hobnail-shaped cells and basally located pleomorphic nuclei without prominent nucleoli were also seen. Panel b shows strong staining for CK7 antigen by immunohistochemical analysis. 
diagnosis of CCAU relies on histological and immunohistochemical analysis. Indeed, results of HE staining and immunohistochemical analysis in our study aligns well with the typical characteristics of CCAU reviewed by Venyo [1].

Primary malignancies of urethra, including squamous cell carcinoma, transitional cell carcinoma and adenocarcinoma, are rare. In women, $90 \%$ of urethral carcinomas are squamous cell carcinoma and transitional cell carcinoma [11]. CCAU, a form of adenocarcinoma is extremely rare. Anatomical distribution of these carcinomas is distinct, which is a key prognostic factor. Squamous cell carcinomas occur predominantly in distal urethra with a better prognosis than adenocarcinomas and transitional cell carcinomas that are more often seen in proximal urethra [10]. After review of current literature, Venyo has shown that patients with CCAU have low 5-year survival rates [1]. The surgical resection (exenteration in women and cystoprostatectomy in men) is the main treatment for CCAU, while radiotherapy is also used in some cases and chemotherapy is less applied $[1,8]$.

\section{Conclusions}

Abnormality in urethrocystoscopy and bloody drainage during the physical exam were not found in this case, in contrast to the findings reported in literature. These unusual features add new knowledge about CCAU which deserves dissemination for improved CCAU diagnosis and management.

\section{Conflict of Interest}

All authors have no competing interests to disclose.

\section{References}

1. Venyo AK. Clear cell adenocarcinoma of the urethra: review of the literature. Int J Surg Oncol. 2015;2015:790235.
2. Tiltman AJ. Primary adenocarcinoma of the female urethra. J Pathol. 1975;117(2):97-99.

3. Ayari Y, Kerkeni W, Znaidi N, Blel A, Bouzouita A, Ayed $\mathrm{H}$, Cherif $\mathrm{M}$, et al. Clear cell adenocarcinoma of the female urethra, mimicking cystocele. Urol Case Rep. 2017;12:26-27.

4. Gogus C, Baltaci S, Orhan D, Yaman O. Clear cell adenocarcinoma of the male urethra. Int J Urol. 2003;10(6):348349.

5. Nakatsuka S, Taguchi I, Nagatomo T, Yamane M, Sugio $\mathrm{K}$, Yoshino R, Oku K, et al. A case of clear cell adenocarcinoma arising from the urethral diverticulum: Utility of urinary cytology and immunohistochemistry. Cytojournal. 2012;9:11.

6. Caplan J, Hartman M, Rooker G. Adenocarcinoma of the female urethra: Clear-cell variant. Radiol Case Rep. 2011;6(3):490.

7. Sadahira T, Maruyama Y, Araki M, Kobayashi Y, Watanabe T, Nasu Y. The Prostate in Female? Clear Cell Adenocarcinoma in a Female Urethral Diverticulum. Urology. 2017;99:e25-e26.

8. Rane SR, Ghodke AN, Vishwasrao S. Clear cell adenocarcinoma of female urethra. J Clin Diagn Res. 2017;11(7):ED01-ED02.

9. Alexiev BA, Tavora F. Histology and immunohistochemistry of clear cell adenocarcinoma of the urethra: histogenesis and diagnostic problems. Virchows Arch. 2013;462(2):193-201.

10. Mehra R, Vats P, Kalyana-Sundaram S, Udager AM, Roh M, Alva A, Pan J, et al. Primary urethral clear-cell adenocarcinoma: comprehensive analysis by surgical pathology, cytopathology, and next-generation sequencing. Am J Pathol. 2014;184(3):584-591.

11. Kim TH, Kim SY, Moon KC, Lee J, Cho JY, Kim SH. Clear cell adenocarcinoma of the urethra in women: distinctive MRI findings for differentiation from nonadenocarcinoma and non-clear cell adenocarcinoma of the urethra. AJR Am J Roentgenol. 2017;208(4):805-811. 